Acta Phys. Hung. B / () -

QUANTUM

ELECTRONICS

\title{
On geometric phases for quantum trajectories
}

\author{
Erik Sjöqvist ${ }^{1, a}$ \\ ${ }^{1}$ Department of Quantum Chemistry, \\ Uppsala University, Box 518, Se-751 20 Uppsala, Sweden \\ Received
}

\begin{abstract}
A sequence of completely positive maps can be decomposed into quantum trajectories. The geometric phase or holonomy of such a trajectory is delineated. For nonpure initial states, it is shown that well-defined holonomies can be assigned by using Uhlmann's concept of parallel transport along the individual trajectories. We put forward an experimental realization of the geometric phase for a quantum trajectory in interferometry. We argue that the average over the phase factors for all quantum trajectories that build up a given open system evolution, fails to reflect the geometry of the open system evolution itself.
\end{abstract}

Keywords: Geometric phase, open systems, quantum trajectories $P A C S$ : 03.65.Vf, 03.65.Yz

\section{Introduction}

Imagine a quantum particle prepared in a pure state. If the state evolves in a unitary fashion so that it remains pure, this particle is said to constitute a closed system. Berry [1] and others [2, 3] 4] have shown that a closed system picks up a phase factor of geometric origin; a geometric phase factor, or quantum holonomy.

But what happens if the system fails to be closed? Is there a natural generalization of the geometric phase to open systems? Considerations of phases of geometric origin for open quantum systems go back to work by Uhlmann [ [5] on holonomy accompanying density operators. Garrison and Wright [ [6] have assigned complex-valued geometric phases to open systems on a phenomenological basis by the use of non-Hermitian Hamiltonians. Ellinas et al. [7] have introduced geometric phases associated with adiabatic evolution of eigenmatrices of the Liouvillian superoperator describing an open system. Gamliel and Freed [ [8] demonstrated that the adiabatic Berry phases may appear also in the presence of open system effects, as phase factors in the off-diagonal elements of the density operator expressed in the instantaneous energy eigenbasis (see also [ 9, 10]). 
More recently, open system geometric phases have been revisited from various perspectives, such as interferometry [11, 12, 13], quantum trajectories [14] 15], phase distributions [16], the adiabatic theorem [ 17], and stochastic Schrödinger evolutions [18]. A reason for this renewed interest is the need to better understand the robustness properties of geometric phases in the presence of open system effects, triggered by the conjectured [19] importance of quantum holonomy for fault tolerant quantum computation. To address the robustness issue, it is commonly assumed that the system evolves under a Markovian master equation. The quantum trajectory approach is specifically designed for the analysis of the robustness of geometric phases to Markovian open system effects (see, e.g., [20, 21]).

In this paper, we focus on the quantum trajectory approach to the open system geometric phase. The purpose is fourfold. First, we wish to reformulate the quantum trajectory approach to the geometric phase on the basis of discrete sets of completely positive maps (CPMs), without invoking continuous master equations. This reformulation is a generalization of previous treatments [14, 15] (see also [22]) in the sense that it yields the master equation in the continuous limit. Secondly, we wish to extend [14] to quantum trajectories that start in a nonpure state. To this end, it seems most natural to use Uhlmann's concept of parallel transport [ [5] along the trajectories. Thirdly, we put forward an experimental procedure to implement the geometric phase for an individual trajectory. Finally, we briefly address whether the geometric phases for individual quantum trajectories can be used to define a meaningful geometric phase for an open system. The paper ends with the conclusions.

\section{Open quantum systems and quantum trajectories}

Consider a quantum system $s$ in contact with some environment $e$, also of quantum nature. Suppose $s+e$ is in the state $\varrho$. Any observable property pertaining only to $s$ is given by the reduced density operator $\rho$ obtained by tracing $\varrho$ over $e$. Let $U\left(t, t_{0}\right)$ be the time evolution operator for $s+e$. Further, let $\left|e_{0}\right\rangle, \ldots,\left|e_{\mu}\right\rangle$ ( $\mu$ could be finite or infinite) be a complete basis for $e$ and suppose that $\varrho=\left|e_{0}\right\rangle\left\langle e_{0}\right| \otimes \rho$ is prepared at $t_{0}$. Then, for an arbitrary $t$, the state of $s$ reads

$$
\rho(t)=\mathcal{E}_{t, t_{0}}(\rho)=\sum_{p=0}^{\mu} E_{p}\left(t, t_{0}\right) \rho E_{p}^{\dagger}\left(t, t_{0}\right),
$$

where $E_{p}\left(t, t_{0}\right)=\left\langle e_{p}\left|U\left(t, t_{0}\right)\right| e_{0}\right\rangle$ are the Kraus operators [ 23] that constitute a Kraus representation of $\mathcal{E}_{t, t_{0}}$. The map $\mathcal{E}_{t, t_{0}}$ is trace preserving and completely positive, i.e., it takes normalized density operators into normalized density operators and all trivial extensions $\hat{1} \otimes \mathcal{E}_{t, t_{0}}$ likewise.

To introduce the concept of quantum trajectories it is convenient to consider $N$ identical copies of the environment. Prepare them in the product state $\left|e_{0}\right\rangle=$ $\left|e_{0}^{0}\right\rangle \otimes \ldots \otimes\left|e_{0}^{N-1}\right\rangle$, with the superscript labeling the copies. Let $s$ interact only with the first copy between $t_{0}$ and $t_{1}$, only with the second copy between $t_{1}$ and $t_{2}$, 
and so on. These interactions are described by the unitary operators $U^{0}\left(t, t_{0}\right), \ldots$, $U^{N-1}\left(t, t_{N-1}\right)$, respectively. It results in the composite map

$$
\rho \rightarrow \mathcal{E}(\rho)=\mathcal{E}_{t_{N}, t_{N-1}} \circ \ldots \circ \mathcal{E}_{t_{1}, t_{0}}(\rho) .
$$

Here, $E_{p}\left(t_{k+1}, t_{k}\right)=\left\langle e_{p}^{k}\left|U^{k}\left(t_{k+1}, t_{k}\right)\right| e_{0}^{k}\right\rangle, p=0, \ldots, \mu$, can be taken as Kraus operators for $\mathcal{E}_{t_{k+1}, t_{k}}, k=0, \ldots, N-1$. In terms of the above $s+e$ picture, the relevant part of the input state at $t_{k}$ is $\left|e_{0}^{k}\right\rangle\left\langle e_{0}^{k}\right| \otimes \rho_{k}$, where $\rho_{0}=\rho$ and $\rho_{k \in[1, N-1]}=$ $\mathcal{E}_{t_{k}, t_{k-1}} \circ \ldots \circ \mathcal{E}_{t_{1}, t_{0}}(\rho)$. A quantum trajectory $\alpha$ is a sequence of (unnormalized) states $\rho \rightarrow \rho_{1}^{\alpha} \rightarrow \ldots \rightarrow \rho_{N}^{\alpha}$, where

$$
\rho_{k}^{\alpha}=E_{\alpha(k)}\left(t_{k}, t_{k-1}\right) \ldots E_{\alpha(1)}\left(t_{1}, t_{0}\right) \rho E_{\alpha(1)}^{\dagger}\left(t_{1}, t_{0}\right) \ldots E_{\alpha(k)}^{\dagger}\left(t_{k}, t_{k-1}\right),
$$

for $k=1, \ldots, N$. Here, $\alpha(l) \in[0, \mu]$ is the 1 th element of a sequence of indexes. The map $\mathcal{E}$ is recovered by summing over all trajectories.

\section{Geometric phase of quantum trajectories}

There is in general an infinite number of possible Kraus representations for a given CPM. For the composite map $\mathcal{E}$ in Eq. (2), different choices of Kraus representations in each step lead to different sets of trajectories. It follows that no physical meaning can be associated to an individual trajectory without imposing some additional physical constraint.

Nevertheless, it is still possible to formally associate a geometric phase or holonomy to an individual quantum trajectory. Here, we wish to do so. In particular, we wish to stress the importance of whether the initial state of the trajectory is pure or nonpure. In the pure case, which has been treated in [14, 22, the standard Pancharatnam connection can be used, while in the nonpure case we need to address the issue of parallel transport of density operators.

Let us first consider the case where the initial state is pure, i.e., $\rho=|\psi\rangle\langle\psi|$. Here, the trajectories may be lifted to sequences of (unnormalized) vectors: $|\psi\rangle \rightarrow$ $\left|\psi_{1}^{\alpha}\right\rangle \rightarrow \ldots \rightarrow\left|\psi_{N}^{\alpha}\right\rangle$, where $\left|\psi_{k}^{\alpha}\right\rangle=E_{\alpha(k)}\left(t_{k}, t_{k-1}\right) \ldots E_{\alpha(1)}\left(t_{1}, t_{0}\right)|\psi\rangle$. The Pancharatnam connection [ 24] yields the geometric phase factor of the trajectory $\alpha$ as

$$
\gamma^{\alpha}=\Phi\left[\left\langle\psi \mid \psi_{N}^{\alpha}\right\rangle\left\langle\psi_{N}^{\alpha} \mid \psi_{N-1}^{\alpha}\right\rangle \ldots\left\langle\psi_{1}^{\alpha} \mid \psi\right\rangle\right],
$$

where $\Phi[z]=z /|z|$ for any nonzero complex number $z$.

When the initial state is nonpure, though, one needs to consider geometric phases for mixed states, since the trajectories then contain nonpure states $\rho, \rho_{1}^{\alpha}, \ldots, \rho_{N}^{\alpha}$ (see Eq. (31)). To deal with this, it seems most natural to use the Uhlmann approach [ [5], since this approach is particularly well adopted to discrete sequences of density operators. Assume that $\rho, \rho_{1}^{\alpha}, \ldots, \rho_{N}^{\alpha}$ are full rank and introduce the amplitudes $W=\sqrt{\rho} V$ and $W_{k}^{\alpha}=\sqrt{\rho_{k}^{\alpha}} V_{k}^{\alpha}, k=1, \ldots, N$, where $V, V_{1}^{\alpha}, \ldots, V_{N}^{\alpha}$ are the unitary operators or 'phases' of the amplitudes. For a given 
but arbitrary $V, V_{1}^{\alpha}$ is fixed by the parallelity condition $W_{1}^{\alpha \dagger} W>0$ and similarly $V_{k+1}^{\alpha}$ is fixed iteratively by $W_{k+1}^{\alpha \dagger} W_{k}^{\alpha}>0$, for $k=1, \ldots, N-1$. In this way, the final unitary operator $V_{N}^{\alpha}$ is uniquely determined by the trajectory $\alpha$ up to the arbitrary phase $V$ of the initial amplitude $W$. Explicitly,

$$
\begin{aligned}
V_{N}^{\alpha}= & \left(\sqrt{\rho_{N}^{\alpha}} \rho_{N-1}^{\alpha} \sqrt{\rho_{N}^{\alpha}}\right)^{-1 / 2} \sqrt{\rho_{N}^{\alpha}} \sqrt{\rho_{N-1}^{\alpha}} \cdots \\
& \times\left(\sqrt{\rho_{2}^{\alpha}} \rho_{1}^{\alpha} \sqrt{\rho_{2}^{\alpha}}\right)^{-1 / 2} \sqrt{\rho_{2}^{\alpha}} \sqrt{\rho_{1}^{\alpha}}\left(\sqrt{\rho_{1}^{\alpha}} \rho \sqrt{\rho_{1}^{\alpha}}\right)^{-1 / 2} \sqrt{\rho_{1}^{\alpha}} \sqrt{\rho} V .
\end{aligned}
$$

To remove the arbitrary phase $V$, define

$$
U^{\alpha}=V_{N}^{\alpha} V^{\dagger}
$$

which is the holonomy of the trajectory $\alpha$. Note that $U^{\alpha}$ reduces to $\gamma^{\alpha}$ in the limit of pure initial states.

\section{Accessing the geometric phase of a quantum trajectory}

As already mentioned, no physical meaning can be associated with a single trajectory and its concomitant geometric phase or holonomy if no further physical constraint is imposed. This is so because there are infinitely many Kraus representations of a given CPM, and therefore infinitely many equivalent ways to decompose the open system evolution into quantum trajectories. Nevertheless, the geometric phase for a single quantum trajectory can be accessed in principle by performing measurements on the environment.

To see this, let us first make some simplifying assumptions. Suppose that $\rho=|\psi\rangle\langle\psi|$ (pure initial state) and that the observables $O_{e}^{k}, k=0, \ldots, N-1$, with eigenstates $\left|e_{0}^{k}\right\rangle, \ldots,\left|e_{\mu}^{k}\right\rangle$, are measured projectively. We further assume that these measurements are performed precisely at $t_{1}, \ldots, t_{N}$, i.e., that the first copy of the environment is measured at $t_{1}$, the second at $t_{2}$, and so on. In the first step, $s+e$ evolves as (ignoring all copies except the first one)

$$
\begin{aligned}
\left|e_{0}^{0}\right\rangle\left\langle e_{0}^{0}|\otimes| \psi\right\rangle\langle\psi| & \rightarrow U^{0}\left(t_{1}, t_{0}\right)\left|e_{0}^{0}\right\rangle\left\langle e_{0}^{0}|\otimes| \psi\right\rangle\langle\psi| U^{0 \dagger}\left(t_{1}, t_{0}\right) \\
& \rightarrow\left|e_{\alpha(1)}^{0}\right\rangle\left\langle e_{\alpha(1)}^{0}\left|\otimes E_{\alpha(1)}\left(t_{1}, t_{0}\right)\right| \psi\right\rangle\langle\psi| E_{\alpha(1)}^{\dagger}\left(t_{1}, t_{0}\right)
\end{aligned}
$$

for the registered outcome $\alpha(1) \in[0, \mu]$. In the second step, the state of $s+e$ similarly evolves as (now ignoring all copies except the second one)

$$
\begin{aligned}
& \left|e_{0}^{1}\right\rangle\left\langle e_{0}^{1}\left|\otimes E_{\alpha(1)}\left(t_{1}, t_{0}\right)\right| \psi\right\rangle\langle\psi| E_{\alpha(1)}^{\dagger}\left(t_{1}, t_{0}\right) \\
& \rightarrow U^{1}\left(t_{2}, t_{1}\right)\left|e_{0}^{1}\right\rangle\left\langle e_{0}^{1}\left|\otimes E_{\alpha(1)}\left(t_{1}, t_{0}\right)\right| \psi\right\rangle\langle\psi| E_{\alpha(1)}^{\dagger}\left(t_{1}, t_{0}\right) U^{1 \dagger}\left(t_{2}, t_{1}\right) \\
& \rightarrow\left|e_{\alpha(2)}^{1}\right\rangle\left\langle e_{\alpha(2)}^{1}\left|\otimes E_{\alpha(2)}\left(t_{2}, t_{1}\right) E_{\alpha(1)}\left(t_{1}, t_{0}\right)\right| \psi\right\rangle\langle\psi| E_{\alpha(1)}^{\dagger}\left(t_{1}, t_{0}\right) E_{\alpha(2)}^{\dagger}\left(t_{2}, t_{1}\right)
\end{aligned}
$$

for the registered outcome $\alpha(2) \in[0, \mu]$. Continuing in this way provides a physical realization of the trajectory $\alpha$. 
We now show that this measurement procedure can be used to experimentally implement $\gamma^{\alpha}$. Suppose $|\psi\rangle\langle\psi|$ is the input state of the internal degree of freedom (e.g. spin) of an ensemble of particles in an ordinary two-beam Mach-Zehnder interferometer. The evolution in Eq. (7) is implemented in one of the interferometer arms by post-selecting those particles for which $\alpha(1)$ is registered. A variable $\mathrm{U}(1)$ phase shift is applied to the other arm. The maximum of the resulting interference fringes is obtained for $\Phi\left[\left\langle\psi\left|E_{\alpha(1)}^{\dagger}\left(t_{1}, t_{0}\right)\right| \psi\right\rangle\right]=\Phi\left[\left\langle\psi_{1}^{\alpha} \mid \psi\right\rangle\right]$. In the next step, the internal state $E_{\alpha(1)}\left(t_{1}, t_{0}\right)|\psi\rangle\langle\psi| E_{\alpha(1)}^{\dagger}\left(t_{1}, t_{0}\right)$ is used as input to a second interferometer in which Eq. (8) is implemented in one arm by post-selecting the outcome $\alpha(2)$. The other arm is again exposed to a variable $\mathrm{U}(1)$ shift, yielding an interference maximum at $\Phi\left[\left\langle\psi\left|E_{\alpha(1)}^{\dagger}\left(t_{1}, t_{0}\right) E_{\alpha(2)}^{\dagger}\left(t_{2}, t_{1}\right) E_{\alpha(1)}\left(t_{1}, t_{0}\right)\right| \psi\right\rangle\right]=$ $\Phi\left[\left\langle\psi_{2}^{\alpha} \mid \psi_{1}^{\alpha}\right\rangle\right]$. Continuing in this way up to $\psi_{N}^{\alpha}$ and back to $\psi$ yields the phase factors $\Phi\left[\left\langle\psi \mid \psi_{N}^{\alpha}\right\rangle\right], \Phi\left[\left\langle\psi_{N}^{\alpha} \mid \psi_{N-1}^{\alpha}\right\rangle\right], \ldots, \Phi\left[\left\langle\psi_{1}^{\alpha} \mid \psi\right\rangle\right]$. By taking the product of these phase factors, we obtain

$$
\Phi\left[\left\langle\psi \mid \psi_{N}^{\alpha}\right\rangle\right] \Phi\left[\left\langle\psi_{N}^{\alpha} \mid \psi_{N-1}^{\alpha}\right\rangle\right] \ldots \Phi\left[\left\langle\psi_{1}^{\alpha} \mid \psi\right\rangle\right]=\Phi\left[\left\langle\psi \mid \psi_{N}^{\alpha}\right\rangle\left\langle\psi_{N}^{\alpha} \mid \psi_{N-1}^{\alpha}\right\rangle \ldots\left\langle\psi_{1}^{\alpha} \mid \psi\right\rangle\right]
$$

which is precisely the geometric phase factor $\gamma^{\alpha}$ in Eq. (4). It should be noted, though, that in order for this procedure to work it is in each step necessary to erase any path information related to the state change of the environment caused by the measurement.

\section{Open system geometric phase}

So far, we have discussed how a geometric phase factor $\gamma^{\alpha}$ or holonomy $U^{\alpha}$ can be assigned to a single quantum trajectory $\alpha$ and how $\gamma^{\alpha}$ can be verified in interferometry. However, since an individual quantum trajectory can differ very much from the original evolution $\mathcal{E}$, it is not obvious in what way $\gamma^{\alpha}$ gives information about the geometry of the path that is generated by $\mathcal{E}$ itself. Such a connection can be established if it is possible to use the geometric phases for the trajectories to assign a well-defined geometric phase for $\mathcal{E}$. Here, we briefly address this issue.

The map $\mathcal{E}$ is recovered by summing over all trajectories $\alpha$. In the case of pure initial state $|\psi\rangle$, it is therefore natural to guess that there is a geometric phase factor $\Gamma_{\mathcal{E}}$ of $\mathcal{E}$ that is the average over the phase factors of the individual quantum trajectories. Explicitly,

$$
\Gamma_{\mathcal{E}}=\sum_{\alpha} p_{\alpha} \gamma^{\alpha}
$$

where $p_{\alpha}=\left\langle\psi_{N}^{\alpha} \mid \psi_{N}^{\alpha}\right\rangle$ is the probability of $\alpha$. Unfortunately, though, this quantity depends on the specific decomposition of $\mathcal{E}$ into trajectories. Thus, unless a specific set of trajectories is singled out (e.g., by repeatedly measuring the environment, as described in the preceding section), no physical meaning can be assigned to $\Gamma_{\mathcal{E}}$. An analogous conclusion in the context of stochastic Schödinger equations has been drawn in $[18]$. 


\section{Conclusions}

The quantum trajectory approach has recently been proposed to tackle the issue of geometric phase [ 14, 22] and quantum holonomy [15] for an open quantum system. The main merit of the approach appears to be its usefulness in the analysis of the robustness of geometric phase based quantum gates to open system effects [20. 21]. Here, we have reformulated the quantum trajectory approach to the geometric phase, on the basis of sequences of completely positive maps.

It has been stressed [14, 22, that the quantum trajectory approach is useful in that it avoids the apparent need to introduce parallel transport of nonpure states. This holds only under the assumption that the trajectory starts in a pure state. We have pointed out that even in the case where the initial state is nonpure it is possible to assign well-defined holonomies by using Uhlmann's concept of parallel transport [ [5] along the quantum trajectories.

The geometric phase of an individual trajectory can be made physical by repeatedly measuring the environment of the system. This opens up the possibility to experimentally implement such phases by measuring post-selectively the environment in one of the arms of a standard Mach-Zehnder interferometry. In this way, we have put forward an iterative procedure to measure the geometric phase of a single quantum trajectory.

Finally, we have addressed the question whether the geometric phases for the trajectories comprising an open system evolution can be used to define the geometric phase of the open system evolution itself. A natural approach is to sum over the individual phase factors weighted by the corresponding probabilities. However, this works only if a specific set of trajectories is singled out by some physical means. It is interesting to note the analogous situation for the geometric phase proposed in [11]. This phase depends on the additional detailed knowledge of the systemenvironment interaction. Thus, neither the approach in [11] nor the averaging over quantum trajectories put forward here, result in a genuine concept of geometric

phase for an open system. It seems more appropriate to use the path of density operator itself as the basis for such a concept [15, 13].

\section{Acknowledgment(s)}

This work was financed in part by the Swedish Research Council.

\section{Notes}

a. E-mail: eriks@kvac.uu.se

\section{References}

1. M.V. Berry, Proc. R. Soc. London Ser. A 392 (1984) 45. 
2. Y. Aharonov and J. Anandan, Phys. Rev. Lett. 58 (1987) 1593.

3. J. Samuel and R. Bhandari, Phys. Rev. Lett. 60 (1988) 2339.

4. N. Mukunda and R. Simon, Ann. Phys. (N.Y.) 228 (1993) 205.

5. A. Uhlmann, Rep. Math. Phys. 24 (1986) 229.

6. J.C. Garrison and E.M. Wright, Phys. Lett. A 128 (1988) 177.

7. D. Ellinas, S.M. Barnett and M.A. Dupertuis, Phys. Rev. A 39 (1989) 3228.

8. D. Gamliel and J.H. Freed, Phys. Rev. A 39 (1989) 3238.

9. K.M. Fonseca Romero, A.C. Aguiar Pinto and M.T. Thomaz, Physica A 307 (2002) 142.

10. I. Kamleitner, J.D. Cresser and B.C. Sanders, Phys. Rev. A 70 (2004) 044103.

11. M. Ericsson, E. Sjöqvist, J. Brännlund, D.K.L. Oi and A.K. Pati, Phys. Rev. A 67 (2003) 020101.

12. J.G. Peixoto de Faria, A.F.R. de Toledo Piza and M.C. Nemes, Europhys. Lett. 62 (2003) 782.

13. D.M. Tong, E. Sjöqvist, L.C. Kwek and C.H. Oh, Phys. Rev. Lett. 93 (2004) 080405 .

14. A. Carollo, I. Fuentes-Guridi, M. Franca Santos and V. Vedral, Phys. Rev. Lett. 90 (2003) 160402.

15. I. Fuentes-Guridi, F. Girelli and E. Livine, Phys. Rev. Lett. 94 (2005) 020503.

16. K.-P. Marzlin, S. Ghose and B.C. Sanders, Phys. Rev. Lett. 93 (2004) 260402.

17. M.S. Sarandy and D.A. Lidar, Phys. Rev. A 73 (2006) 062101.

18. A. Bassi and E. Ippoliti, Phys. Rev. A 73 (2006) 062104.

19. P. Zanardi and M. Rasetti, Phys. Lett. A 264 (1999) 94.

20. J.K. Pachos and A. Beige, Phys. Rev. A 69 (2004) 033817.

21. L.-X. Cen and P. Zanardi, Phys. Rev. A 70 (2004) 052323.

22. A. Carollo, Mod. Phys. Lett. A 20 (2005) 1635.

23. K. Kraus, States, Effects, and Operations, Springer, Berlin, 1983.

24. S. Pancharatnam, Proc. Ind. Acad. Sci. A 44 (1956) 247. 